\title{
Glycine Is a Predictor for a Suppressive Effect of Fucoxanthinol on Colonosphere Formation Under Hypoxia
}

\author{
MASARU TERASAKI ${ }^{1,2}$, YUKI OGAWA ${ }^{1}$, TETSUYA ENDO $^{1}$, HAYATO MAEDA $^{3}$, \\ JUNICHI HAMADA ${ }^{2,4}$, KAZUMI OSADA ${ }^{5}$, KAZUO MIYASHITA ${ }^{6}$ and MICHIHIRO MUTOH ${ }^{7}$ \\ ${ }^{1}$ School of Pharmaceutical Sciences, ${ }^{2}$ Cancer Prevention Laboratories, ${ }^{4}$ School of Nursing and Social Services, and \\ ${ }^{5}$ School of Dentistry, Health Sciences University of Hokkaido, Tobetsu, Japan; \\ ${ }^{3}$ Department of Applied Biosciences, Faculty of Agriculture and Life Science, Hirosaki University, Hirosaki, Japan; \\ ${ }^{6}$ Laboratory of Biofunctional Material Chemistry, Division of Marine Bioscience, \\ Graduate School of Fisheries Sciences, Hokkaido University, Hakodate, Japan; \\ ${ }^{7}$ Epidemiology and Preventions Group, Center for Public Health Sciences, National Cancer Center, Tokyo, Japan
}

\begin{abstract}
Background/Aim: Fucoxanthinol (FxOH), a metabolite of fucoxanthin, is known to inhibit tumorigenicity of human colorectal cancer stem cells (CCSCs) and their sphere formation. Hypoxic conditions and hypoxia-inducible factors (HIFs) are essential to maintain the stemness of CCSCs. We investigated effects of $\mathrm{FxOH}$ on sphere formation, intercellular energy metabolites in colonospheres formed from human colorectal HT-29 cells under hypoxic conditions. Results: FxOH at $50 \mu M$ suppressed HIFla expression and activation of integrin, mitogen-activated protein kinase (MAPK), Wingless/integrated (WNT) and signal transducer and activator of transcription (STAT) signals. Moreover, expression of epithelial-mesenchymal transition- and apoptosis-related proteins in the colonospheres was lowered by $\mathrm{FxOH}$. The level of glycine was reduced in hypoxic colonospheres under $\mathrm{FxOH}$ treatment. Conclusion: FxOH attenuated the sphere formation of hypoxic colonospheres, in part, by suppressing HIFl $\alpha$ expression. Glycine could be a potential predictor for the activity of agents that inhibit sphere formation by hypoxic colonospheres.
\end{abstract}

Colorectal cancer (CRC) is one of the most common causes of cancer-related death worldwide. Thus, effective preventive strategies against CRC are urgently required (1). Many preventive trials for $\mathrm{CRC}$, including risk avoidance and

Correspondence to: Dr Masaru Terasaki, School of Pharmaceutical Sciences, Health Sciences University of Hokkaido, 1757 Kanazawa, Ishikari-Tobetsu, Hokkaido 061-0293, Japan. E-mail: terasaki@ hokuiryo-u.ac.jp

Key Words: Fucoxanthinol, colonosphere, colorectal cancer stem cell, hypoxia, metabolite. functional food intervention, have failed to obtain satisfactory results (2). Development of CRC stem cells (CCSCs) within normal colorectal mucosa may induce resistance to test agents in such trials.

CCSCs are known to comprise only a minor subpopulation in those tissues, but have been regarded as pivotal cells required for cancer development, recurrence and distant metastasis. CCSCs have multiple properties, such as self-renewal, differentiation, sphere formation, and tumorigenicity $(3,4)$. Human CRC tissues are hypoxic (less than $5 \% \mathrm{O}_{2}$ ) with poor vascularization.

Hypoxia-inducible factors (HIFs) are transcriptional activators that induce conditional expression of hypoxia-related protein regarding CCSC formation and cell surface markers (5, 6). HIFs play an important role in drug resistance, angiogenesis, apoptosis, cell-cycle arrest, epithelial-mesenchymal transition (EMT), genome instability, inflammation, invasiveness, metastasis and metabolism alteration (7-11).

Intracellular metabolites such as amino and carboxylic acids could be useful indicators that represent complicated genomic and proteomic responses after various stimuli. Data on candidate metabolites as invasive and non-invasive markers in patients with CRC and in animal research have been accumulated (12-14). However, little information has been gathered on cellular metabolism in relation to indicator metabolites representing the cellular condition of CCSCs under hypoxia.

Fucoxanthin is a major marine xanthophyll that has been shown to provide several health benefits to humans. Edible brown algae such as Undaria pinnatifida (wakame) are particularly excellent sources of fucoxanthin among Japanese algal foods $(15,16)$. Several researchers have reported the anticancer effect of fucoxanthin in rodent models of CRC $(17,18)$. Moreover, fucoxanthin strongly induces cell growth arrest and apoptosis in human CRC cells (19-21). 
Among the metabolites of fucoxanthin, fucoxanthinol $(\mathrm{FxOH})$ may play a role against cancer cells and is also a promising candidate for human cancer chemoprevention. After oral administration of oil-fried wakame or fucoxanthin concentrate in humans, high levels of $\mathrm{FxOH}$ (Figure 1) and low levels of cis- $\mathrm{FxOH}$ are detected in human plasma $(22,23)$. FxOH has the potential to suppress the growth of cultured cancer cells isolated from human CRC tissue (24).

Colonospheres (CS) of CRC cells are a good model to investigate CCSC because they contain abundant CCSCs, and show sphere-reconstruct and tumorigenic capacities (3, $4,24)$. $\mathrm{FxOH}$ was found to strongly induce the apoptosis of CS formed from human CRC HT-29 cells and reduce tumors generated by $\mathrm{CS}$ (25). $\mathrm{FxOH}$ also attenuated sphere formation and EMT, inhibited the activation of integrin, mitogen-activated protein kinase (MAPK), and signal transducer and activator of transcription (STAT) signaling, induced apoptosis, and altered metabolite profiles in CS formed from HT-29 and HCT116 human CRC cells under normoxia (M. Terasaki et al.; unpublished data). In the study, it was demonstrated that glycine and succinic acid were representative indicators of suppression of EMT and sphere formation in CS. However, little information regarding the anti-metabolic effect of $\mathrm{FxOH}$ on CCSCs or CS under hypoxic conditions is available.

In the present study, we investigated the effects on sphere formation, and the molecular mechanisms of $\mathrm{FxOH}$ treatment on CS formed from HT-29 cells under hypoxic conditions. In addition, we examined the metabolic profiles of $\mathrm{CS}$ under $\mathrm{FxOH}$ treatment.

\section{Materials and Methods}

Chemicals and cell culture. HT-29 human CRC cells were obtained from the American Type Culture Collection (Manassas, VA, USA). The cells were maintained on a culture dish in Dulbecco's modified Eagle's medium (DMEM) supplemented with $10 \%$ heat-inactivated fetal bovine serum (FBS), $4 \mathrm{mM} \mathrm{L}$-glutamine, 40,000 U/1 penicillin, and $40 \mathrm{mg} / \mathrm{l}$ streptomycin in humidified normoxic air $\left(20 \% \mathrm{O}_{2}\right.$ and $5 \% \mathrm{CO}_{2}$ ). All-trans-FxOH (purity, $\geq 98 \%$ ) was prepared by Dr. Hayato Maeda (Hirosaki University, Japan) (Figure 1). Epidermal growth factor (EGF), basic fibroblast growth factor (bFGF) and DMEM/F12 medium were purchased from Wako Pure Chemicals (Osaka, Japan). B27 supplemental solution was obtained from Miltenyi Biotec, Inc. (Auburn, CA). All the other chemicals and solvents used in the study were of analytical grade, unless otherwise mentioned.

CS formation. Adherent parental HT-29 cells were collected by trypsinization, washed twice with PBS, suspended in stem cell medium (SCM) composed of DMEM/F12 medium, $20 \mathrm{ng} / \mathrm{ml}$ EGF, $10 \mathrm{ng} / \mathrm{ml} \mathrm{bFGF}, 0.2 \%$ B27 and an antibiotic-anti-mycotic agent, seeded at a density of 30,000 cells/ml SCM in $10-\mathrm{cm}$ or $24-w e l l$ ultra-low attachment plates (Corning, NY, USA) and incubated for 2 days at $37^{\circ} \mathrm{C}$ in humidified air under hypoxic $\left(1 \% \mathrm{O}_{2}\right.$ and $5 \%$

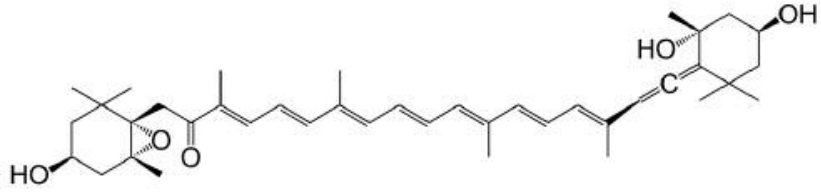

Figure 1. The structure of fucoxanthinol.

$\left.\mathrm{CO}_{2}\right)$ or normoxic conditions $\left(20 \% \quad \mathrm{O}_{2}\right.$ and $\left.5 \% \quad \mathrm{CO}_{2}\right)$. All experiments utilizing CS described below were performed using CS grown for 2 days.

Secondary CS formation. HT-29 CS formed under hypoxic conditions and normoxic conditions for 2 days were trypsinized and seeded at a daily of 30,000 cells $/ \mathrm{ml} \mathrm{SCM}$ into 24 wells of an ultralow attachment plate and incubated subsequently for 2 days under hypoxic conditions or normoxic conditions. After 2 days, the CS were harvested and trypsinized. Viable cells in CS were counted using the trypan blue exclusion method.

Analysis of effects of $\mathrm{FxOH}$ on formation of CS. Parental HT-29 cells were allowed to form CS by culture in a 24-well ultra-low attachment plate for 2 days under hypoxic conditions or normoxic conditions. After 2 days, a total of 2-10 $\mathrm{mM}$ FxOH reconstituted in dimethyl sulfoxide (DMSO) was applied to the cell medium at a final concentration of 10 to $50 \mu \mathrm{M}(0.5 \mathrm{v} / \mathrm{v} \%)$, or vehicle alone (DMSO) was applied to hypoxic and normoxic CS which were then further incubated for $24 \mathrm{~h}$ under hypoxic conditions and normoxic conditions, respectively. The cells were harvested and trypsinized after incubation for $24 \mathrm{~h}$. Viable cells in CS were counted using the trypan blue exclusion method.

Western blot. Antibodies against $\beta$-actin, E-cadherin, $N$-cadherin, caspase-3, phospho (p)-focal adhesion kinase (FAK) $\left(\mathrm{Tyr}^{397}\right.$ ), HIF1 $\alpha$ and vimentin were obtained from GeneTex (Irvine, CA, USA). $p$-Paxillin $\left(\mathrm{Tyr}^{31}\right.$ ) antibody was purchased from $\mathrm{R} \& \mathrm{D}$ Systems (Minneapolis, MN, USA). CD44 and p53 antibodies were obtained from Thermo Fisher Scientific (Waltham, MA, USA). Epithelial cell adhesion molecule (EpCAM) and integrin $\beta 1$ antibodies were purchased from EXBIO (Prague, Czech Republic). pAKT $\left(\mathrm{Ser}^{473}\right), \quad \beta$-catenin, $\mathrm{p} \beta$-catenin (Ser31/37/Thr42), cyclin D1 and peroxisome proliferatoractivated receptor $\gamma(\operatorname{PPAR} \gamma)$ antibodies, and pERK1/2 pathway and p-STAT antibody sampler kits were obtained from Cell Signaling Technology (Danvers, MA, USA). The CS formed under hypoxic and normoxic conditions were seeded in $10-\mathrm{cm}$ ultra-low attachment plates at a density of 30,000 cells $/ \mathrm{ml} \mathrm{SCM}$, treated with $\mathrm{FxOH}$ and collected after 4-24 h under subsequent hypoxic conditions and normoxic conditions, respectively. The CS were recovered, and then lysed in a lysis buffer for preparation of whole-cell lysates. For analysis of HIF1 $\alpha$ expression, the CS were trypsinized and suspended in $50 \mu \mathrm{l}$ of isotonic buffer containing $10 \mathrm{mM}$ Tris- $\mathrm{HCl}, \mathrm{pH} 7.4,10 \mathrm{mM} \mathrm{NaCl}$ and $1.5 \mathrm{mM}$ $\mathrm{MgCl}_{2}$ on ice for $30 \mathrm{~min}$. The cells were homogenized by using pipette with a $200 \mu \mathrm{l}$ disposable tip. The nuclei in the disrupted cells were then recovered at $800 \times g$ centrifugation for $10 \mathrm{~min}$ and lysed in lysis buffer. Fifty micrograms of whole-cell or 
A
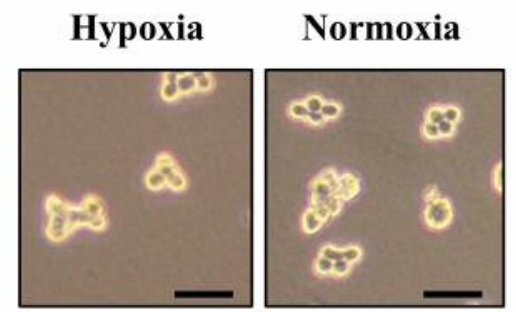

C

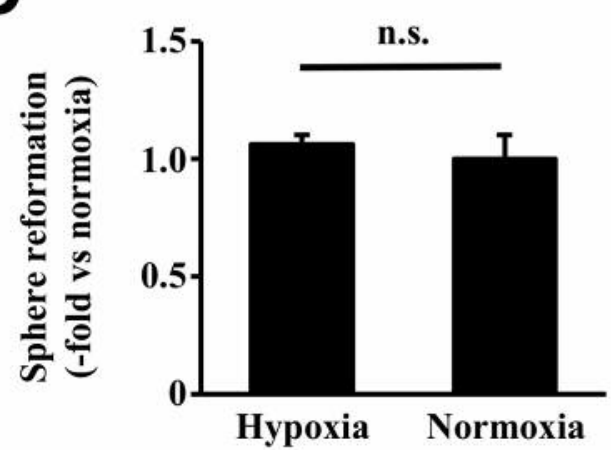

B

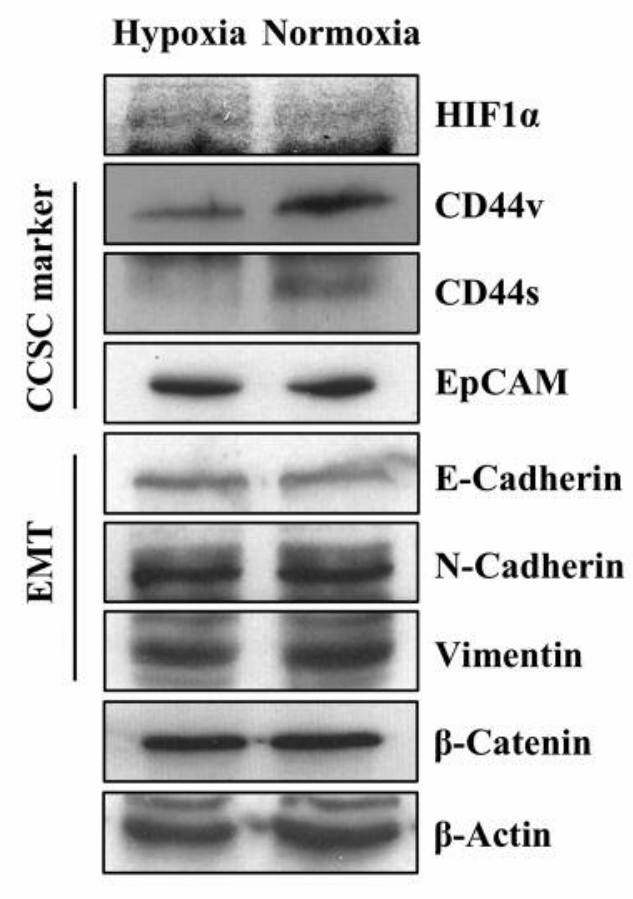

Figure 2. The change in protein expression of colorectal cancer stem cell (CCSC) and epithelial-mesenchymal transition (EMT) markers between hypoxic and normoxic conditions in colonospheres (CS) formed from parental HT-29 cells. CS were formed by treatment with stem cell medium for 2 days under hypoxic and normoxic conditions. A: Photographs of HT-29 CS were taken under phase-contrast microscopy. Bar, $100 \mu$ m. B: CS under hypoxic and normoxic conditions were collected and the same amount of their protein was subjected to western blot. C: The primary CS prepared from HT-29 cells for 2 days under hypoxic and normoxic conditions were respectively dissociated into a single-cell suspension and subsequently subjected to secondary sphere formation assay under hypoxic and normoxic conditions. After 2 days, the sphere-reforming activity of HT-29 CS was determined by cell count assay with the trypan blue exclusion method. Values are means \pm SE (n=3). n.s.: Not significantly different by Welch's t-test. HIF1 $\alpha$ : Hypoxia-inducible factor 1 $\alpha$; EpCAM: epithelial cellular adhesion molecule.

nuclear proteins were separated on sodium dodecyl sulfatepolyacrylamide gel electrophoresis and transferred to a polyvinylidene fluoride membrane. The polvinylidene fluoride membrane was blocked with $1 \%$ bovine serum albumin in Trisbuffered saline/Tween-20 at room temperature and probed with each of the primary antibodies in the blocking buffer following the manufacturer's instructions, overnight at $4^{\circ} \mathrm{C}$. The membranes were treated with horseradish peroxidase conjugated anti-mouse or anti-rabbit secondary antibodies and proteins were detected by chemiluminescence reagents.

Gas chromatography-mass spectrometry (GC-MS) analysis. CS treated with $\mathrm{FxOH}$ under hypoxic conditions or normoxic conditions were collected from culture plates, trypsinized, and washed with PBS. The pelleted cells were re-suspended in $50 \mu \mathrm{l}$ of cold PBS, $0.2 \mu \mathrm{g}$ of 2-isopropylmalic acid was added as an internal standard, and then the suspensions were disrupted by sonication for $5 \mathrm{~s}$ on ice. Total protein contents were measured by the Bradford method. Metabolites from the measured amount of proteins were extracted with $250 \mu \mathrm{l}$ of $\mathrm{CH}_{3} \mathrm{OH} / \mathrm{CHCl}_{3} /$ distilled water $(2.5: 1: 1, \mathrm{v} / \mathrm{v} / \mathrm{v})$, centrifuged at $16,000 \times g$ for $5 \mathrm{~min}$ and the upper phase was washed with $200 \mu 1$ of distilled water. The extracts obtained were evaporated to dryness. The residues were derivatized by methoxyamine hydrochloride and $\mathrm{N}$-methyl$\mathrm{N}$ (trimethylsilyl)-trifluoroacetate. GC-MS was carried out using a GCMS-QP5000 system (Shimadzu, Kyoto, Japan) equipped with an Rxi-5ms column ( $30 \mathrm{~m} \times 0.25 \mathrm{~mm}$ i.d., film thickness 0.25 $\mu \mathrm{m}$; RESTEK Co. Ltd., GmbH, Bad Homburg, Germany) and data software (CLASS 5000). The carrier gas was helium at 0.5 $\mathrm{ml} / \mathrm{min}(15.7 \mathrm{kPa})$ and samples $(1 \mu \mathrm{l})$ were injected in split-ratio mode (split ratio, $33 \%$ ). Column temperature was initially $80^{\circ} \mathrm{C}$ for $2 \mathrm{~min}$, then raised to $330^{\circ} \mathrm{C}$ at $4^{\circ} \mathrm{C} / \mathrm{min}$, and then held at $330^{\circ} \mathrm{C}$ for $8 \mathrm{~min}$. Interface and source temperatures were at 250 and $230^{\circ} \mathrm{C}$, respectively. Identification was confirmed by authentic standards and comparing the spectra of the single components with those stored in the acquisition system library. All metabolite contents were expressed in $\mathrm{pmol} / \mu \mathrm{g}$ total protein content.

Statistical analysis. All experiments were performed at least in triplicate and are presented as representative data. Significant differences were determined by Welch's $t$-test and one-way ANOVA with post-hoc Tukey-Kramer test for multiple comparisons. Statistically significant differences were accepted at $p<0.05$. 


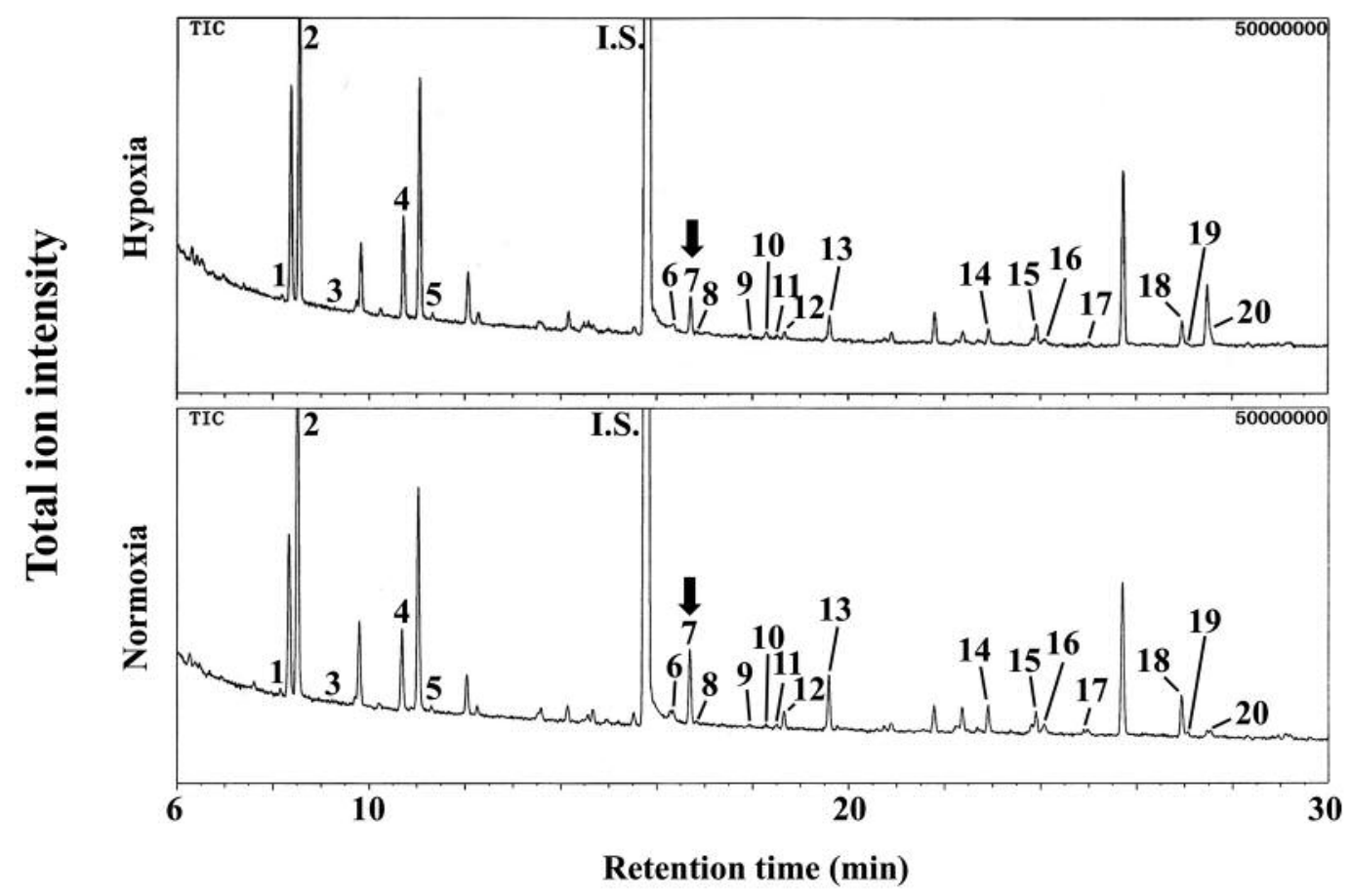

Figure 3. The change of gas chromatography-mass spectrometry (GC-MS) total ion chromatograms (TIC) of metabolites in colonospheres (CS) formed from parental HT-29 cells between hypoxic conditions and normoxic conditions. CS from HT-29 cells under hypoxic and normoxic conditions were collected and their metabolites were analyzed by GC-MS. Peak 7 indicates glycine (arrow). The data for cellular metabolites corresponding to each peak number are shown in Table I. GC-MS conditions are given in the Materials and Methods section. I.S.: Internal standard (2-isopropylmalic acid).

\section{Results}

Stemness and metabolite profile of CS under different oxygen conditions. Protein expressions of CS formed from HT-29 cells under hypoxic and normoxic conditions were determined by western blot (Figure 2A and B). HIF1 $\alpha$ expression was increased under hypoxic conditions in comparison with normoxic conditions. On the other hand, CCSC cell surface markers, variant (CD44v) and standard (CD44s) CD44 forms were decreased in CS under hypoxic conditions compared with those under normoxic conditions. Molecular markers, such as a CCSC marker EpCAM, $\beta$ catenin, and key proteins representing the EMT phenotype (E-cadherin, $N$-cadherin and vimentin) did not change between CS under hypoxia and normoxia. Moreover, little difference was observed between the two conditions regarding the sphere-reforming activity of CS (Figure 2C).

We next investigated the metabolite profiles of CS under hypoxic conditions and normoxic conditions using GC-MS (Figure 3). The quantitative data of all 20 metabolites from CS analyzed under these two conditions are presented in Table I. The level of one amino acid, namely glycine, was significantly lower in CS under hypoxia compared with normoxia. Almost all other metabolites were reduced under hypoxic conditions compared with normoxic conditions, but without statistical difference.

Antiproliferative effect of $\mathrm{FxOH}$ on $\mathrm{CS}$ under different oxygen conditions. Treatment with 10, 20 and $50 \mu \mathrm{M} \mathrm{FxOH}$ suppressed the sphere formation of CS under both hypoxic and normoxic conditions in a dose-dependent manner (Figure $4 \mathrm{~A}$ and $\mathrm{B}$ ). The vehicle (DMSO) alone showed no effect on cell proliferation.

Change of metabolite profile of $\mathrm{CS}$ induced by $\mathrm{FxOH}$ under different oxygen conditions. Metabolite profiles of CS formed from HT-29 cells under hypoxic conditions or normoxic conditions were examined using GC-MS (Figure 5). The quantitative data of all 20 analyzed metabolites from CS treated with $50 \mu \mathrm{M}$ FxOH under the two oxygen conditions are presented in Table II. Glycine was significantly decreased in CS under both hypoxic conditions and normoxic conditions. Five amino acids, proline, serine, threonine, aspartic acid, glutamic acid, and succinic acid (a carboxylic acid involved in the tricarboxylic acid cycle) were found at significantly lower levels in CS under normoxia. No significant change was observed in other metabolites under both oxygen conditions. 


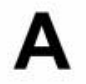

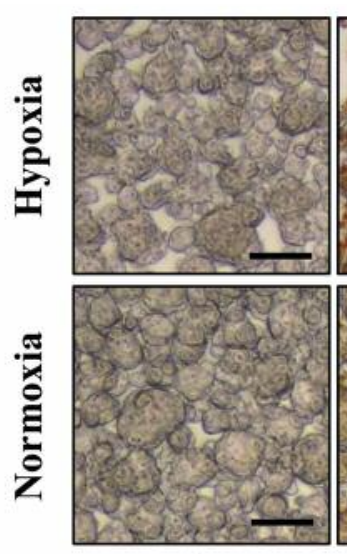

$\mathbf{0}$

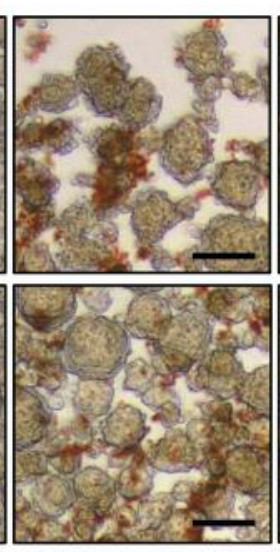

10
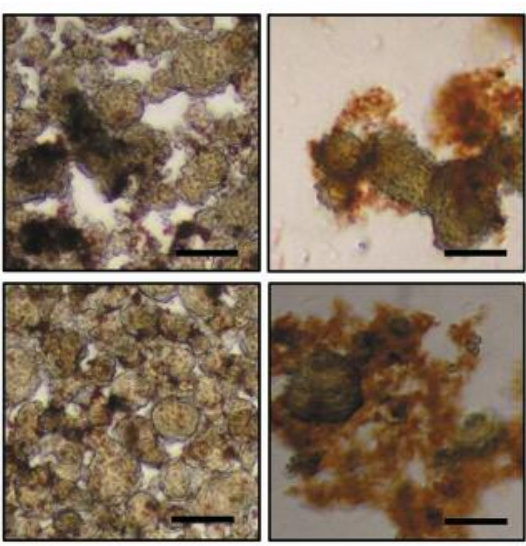

20

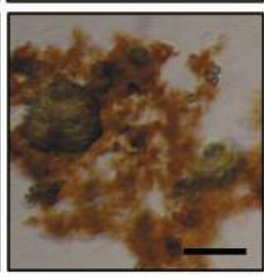

50

\section{FxOH $(\mu \mathrm{mol} / \mathrm{l})$}
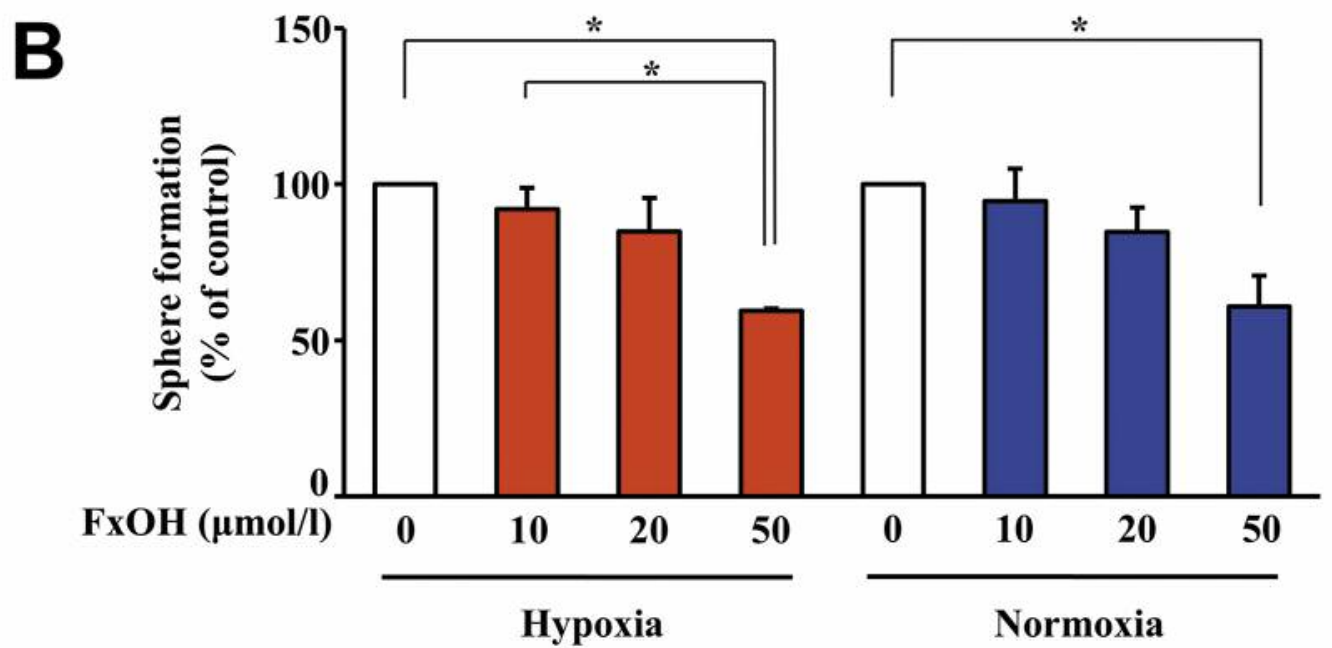

Figure 4. Antiproliferative effects of fucoxanthinol ( $F x O H)$ in colonospheres (CS) of HT-29 cells under hypoxic and normoxic conditions. CS formed from HT-29 cells for 2 days under hypoxic and normoxic conditions were treated with 10-50 $\mu \mathrm{M}$ of FxOH for an additional 24 h under hypoxic and normoxic conditions. A: Photographs of HT-29 CS treated with FxOH for 1 day were taken using phase-contrast microscopy. Bar, $100 \mu$ m. Brown masses show FxOH deposits. B: Sphere-forming activity of HT-29 CS was determined by cell count assay with the trypan blue exclusion method. Values are means \pm SE $(n=3)$. *Significantly different at $p<0.05$ by one-way ANOVA with post-hoc Tukey-Kramer test.

Expression of molecules related to $\mathrm{FxOH}$ treatment under hypoxic conditions. To evaluate the protein levels involved in growth, EMT and apoptosis in CS under hypoxic conditions, we performed western blot assay. $\mathrm{FxOH}$ downregulated HIF1 $\alpha$ expression from 8 to $24 \mathrm{~h}$ in HT-29 CS. In addition, FxOH reduced the activation of molecules involved in integrin, MAPK, WNT and STAT signaling, and EMT. Twenty- four hours' treatment with $\mathrm{FxOH}$ abrogated p53 expression and activation of caspase-3, which was detected in the p17 and p19 active forms. In particular, $\mathrm{FxOH}$ reduced PPAR $\gamma$ expression as early as $4 \mathrm{~h}$ (Figure 6).
Time-dependent change of glycine level in CS under hypoxic conditions after FxOH treatment. The glycine level was measured in HT-29 CS treated with $50 \mu \mathrm{M}$ FxOH for 4, 8 and $24 \mathrm{~h}$. FxOH significantly increased the glycine level from 4 to $8 \mathrm{~h}$ after treatment, but reduced it from 8 to $24 \mathrm{~h}$ (Figure 7).

\section{Discussion}

Our study suggests that $\mathrm{FxOH}$ inhibited sphere formation in HT-29 CS under hypoxic conditions mediated by suppressing HIF1 $\alpha$ expression, integrin, MAPK, WNT and STAT 


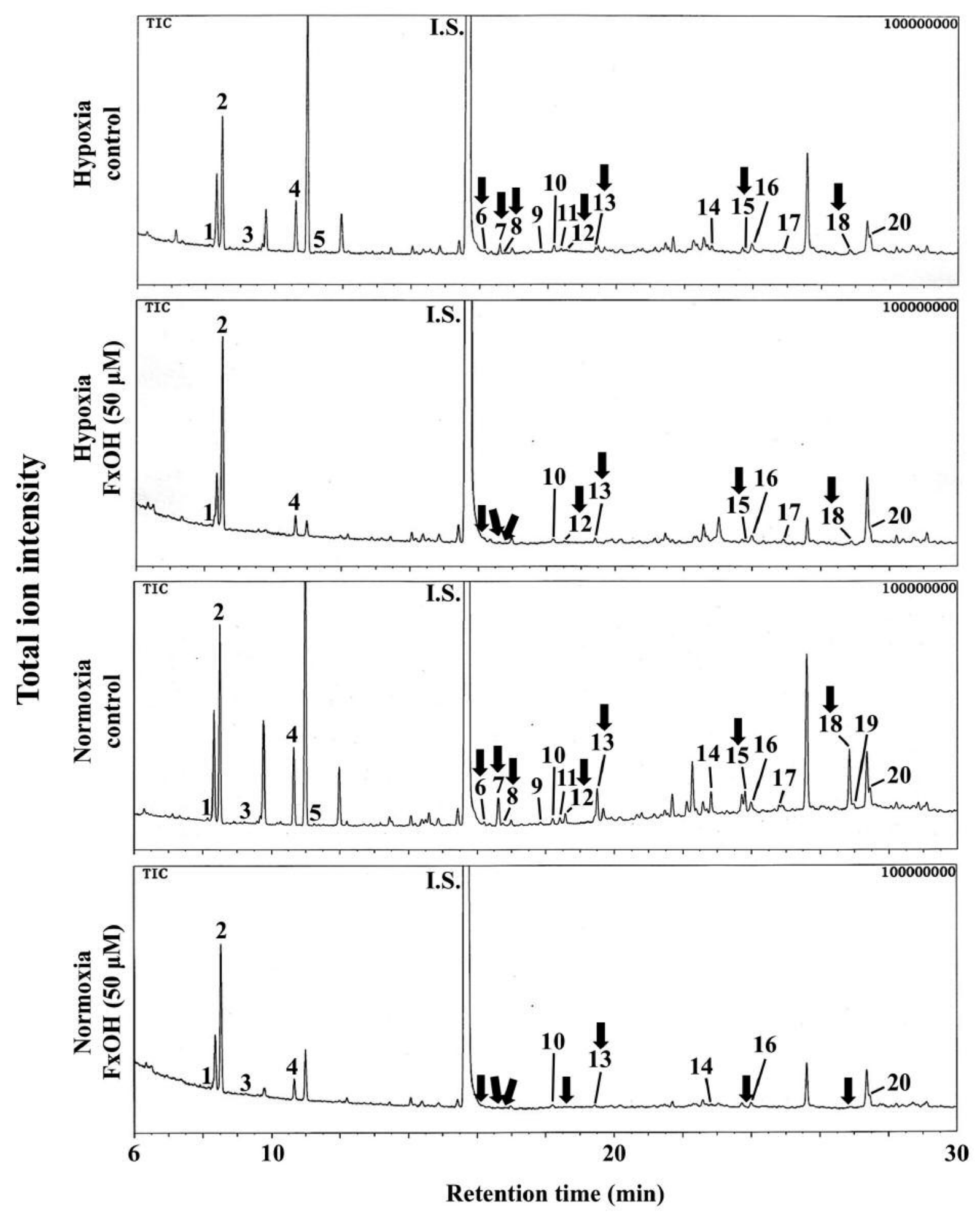

Figure 5. The change of gas chromatography-mass spectrometry (GC-MS) total ion chromatograms (TIC) of metabolites in colonospheres (CS) formed from HT-29 cells treated with fucoxanthinol $(\mathrm{FxOH})$ under hypoxic or normoxic conditions. CS formed from HT-29 cells for 2 days under hypoxic or normoxic conditions were treated with 10-50 $\mu \mathrm{M}$ of $\mathrm{FxOH}$ for an additional $24 \mathrm{~h}$ under hypoxic or normoxic conditions. Their metabolites were then analyzed by GC-MS. Peaks 6, 7, 8, 12,13, 15 and 18 indicate glycine, succinic acid, serine, threonine, aspartic acid and glutamic acid, respectively (arrows). The data for cellular metabolites corresponding to each peak number are shown in Table II. GC-MS conditions are given in the Materials and Methods section. I.S.: Internal standard (2-isopropylmalic acid). 
A

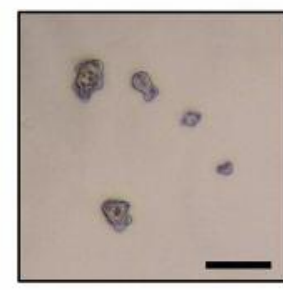

0

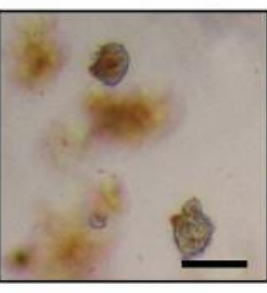

4

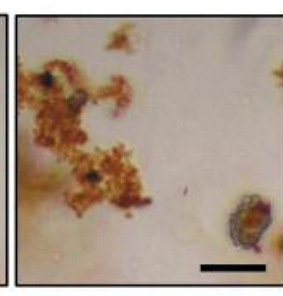

8

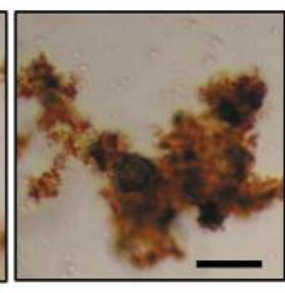

24

Time (h)

B

Time (h)

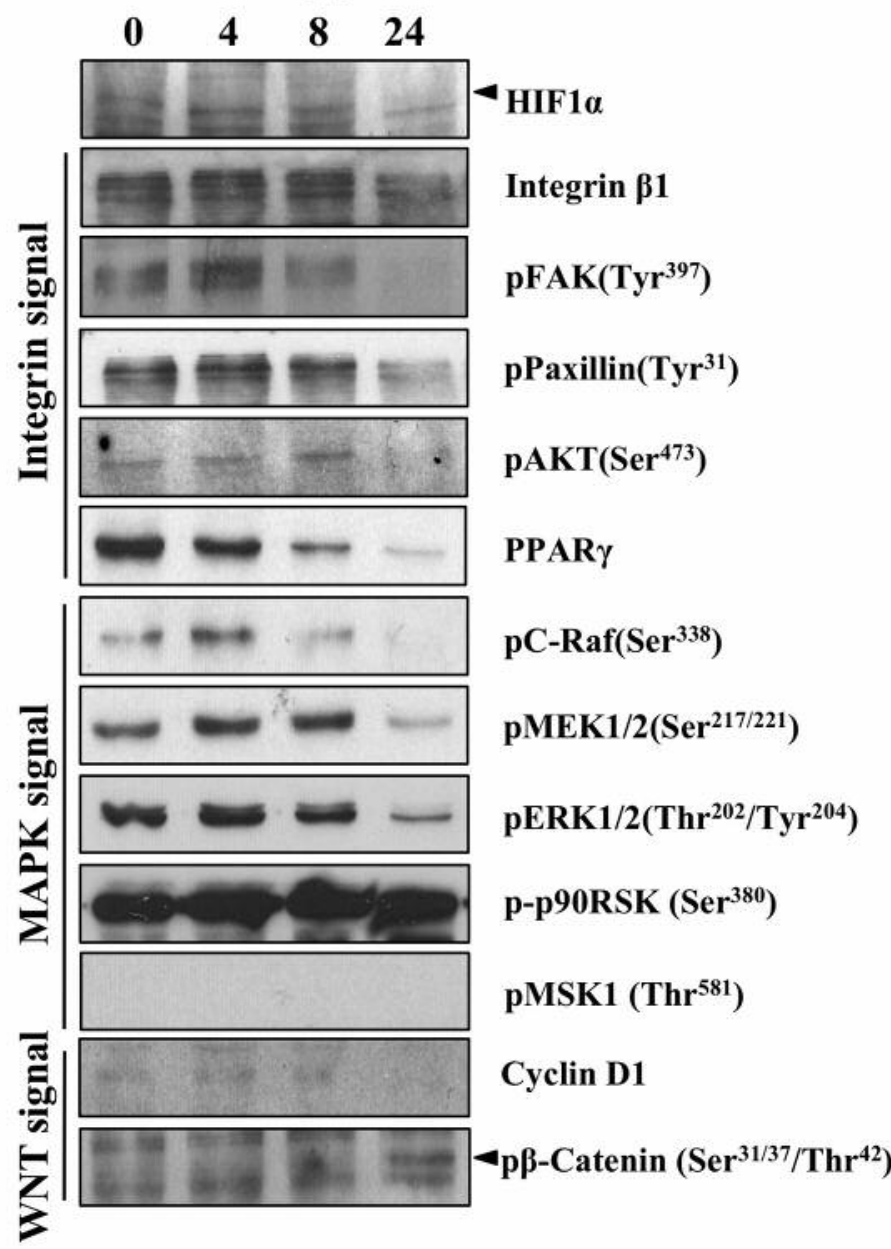

Time (h)
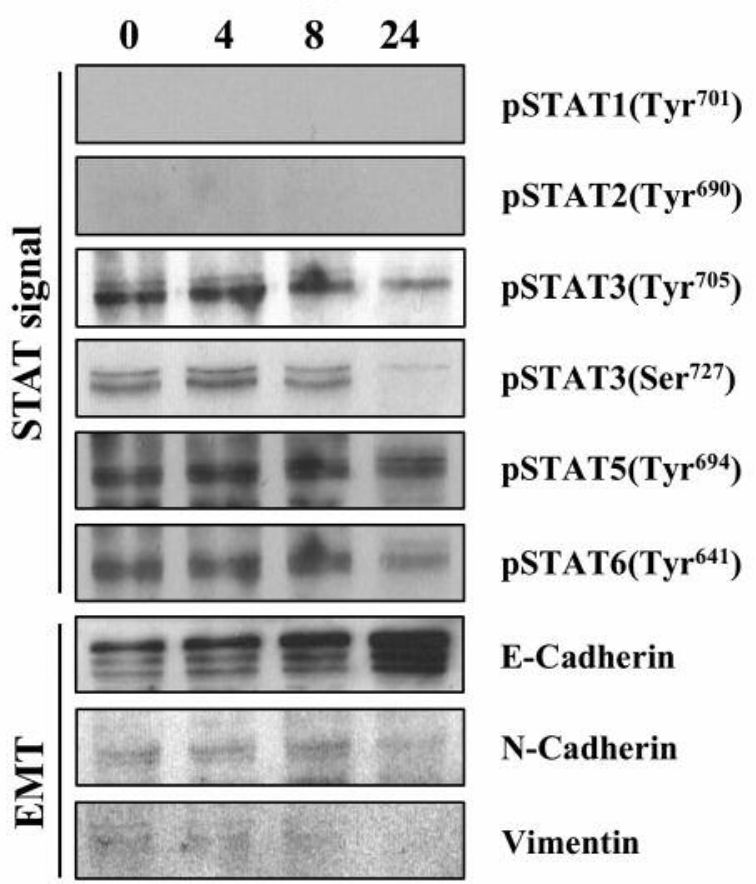

E-Cadherin

N-Cadherin

\section{Vimentin}

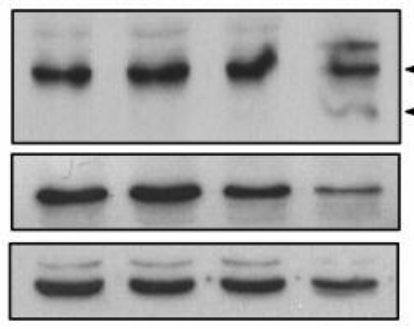

Pro-caspase-3

Cleaved p17/p19

p53

\section{$\beta$-Actin}

Figure 6. Western blot analysis of colonospheres (CS) formed from HT-29 cells with fucoxanthinol (FxOH) treatment under hypoxic conditions. CS formed from HT-29 cells for 2 days under hypoxic conditions were treated with $50 \mu M$ of FxOH for 4, 8 and 24 additional h under subsequent hypoxic conditions. A: Photograph of CS from HT-29 cells treated with FxOH under hypoxic conditions were taken using phase-contrast microscopy. Brown masses show FxOH deposits. Bar, $100 \mu \mathrm{m}$. B: HT-29 CS were collected and the same amount of their protein was subjected to western blot.

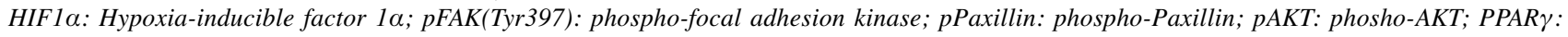
peroxisome proliferator-activated receptor $\gamma ; p C$-Raf: phospho-C-Raf; pMEK: phospho-mitogen-activated protein/extracellular signal-regulated kinase (ERK) kinase; p-p90RSK: phospho- 90 ribosomal S6 protein kinase; pMSK1: phospho-mitogen- and stress-activated protein kinase-1; $\beta$ Catenin: phospho- $\beta$-Catenin; pSTAT: phospho-signal transducer and activator of transcription. 
Table I. Metabolite profiles in colonospheres under hypoxic and normoxic conditions.

\begin{tabular}{|c|c|c|c|}
\hline \multirow[t]{2}{*}{$\begin{array}{l}\text { Peak } \\
\text { no. }{ }^{\text {a }}\end{array}$} & \multirow[t]{2}{*}{ Compound } & \multicolumn{2}{|c|}{$\begin{array}{l}\text { Metabolite, } \mathrm{pmol} / \mu \mathrm{g} \\
\text { total protein content }\end{array}$} \\
\hline & & Hypoxia & Normoxia \\
\hline & Amino acids & & \\
\hline 3 & Valine & $0.8 \pm 0.8^{b}$ & $3.4 \pm 2.0$ \\
\hline 5 & Leucine & $2.9 \pm 0.6$ & $3.4 \pm 0.6$ \\
\hline 6 & Proline & $3.0 \pm 0.9$ & $8.3 \pm 1.2$ \\
\hline 7 & Glycine & $9.3 \pm 2.6 *$ & $21.9 \pm 3.1$ \\
\hline 11 & Alanine & $0.2 \pm 0.2$ & $0.4 \pm 0.4$ \\
\hline 12 & Serine & $2.1 \pm 0.2$ & $2.7 \pm 0.8$ \\
\hline 13 & Threonine & $5.1 \pm 1.6$ & $8.7 \pm 2.3$ \\
\hline 15 & Aspartic acid & $2.9 \pm 1.3$ & $4.3 \pm 0.9$ \\
\hline 18 & Glutamic acid & $4.7 \pm 1.6$ & $9.2 \pm 1.1$ \\
\hline \multirow[t]{2}{*}{19} & Phenylalanine & $0.5 \pm 0.5$ & $1.2 \pm 0.4$ \\
\hline & Dicarboxylic acids (TCA cycle) & & \\
\hline 8 & Succinic acid & $0.2 \pm 0.2$ & $0.8 \pm 0.2$ \\
\hline 9 & Fumaric acid & $0.4 \pm 0.4$ & $1.2 \pm 0.9$ \\
\hline \multirow[t]{2}{*}{14} & Malic acid & $3.7 \pm 0.6$ & $4.5 \pm 1.5$ \\
\hline & Carboxylic acids/other & & \\
\hline 1 & Pyruvic acid & $1.8 \pm 0.9$ & $2.1 \pm 0.8$ \\
\hline 2 & Propionic acid & $103.9 \pm 15.0$ & $83.5 \pm 20.3$ \\
\hline 4 & Butyric acid & $34.6 \pm 1.3$ & $36.4 \pm 3.9$ \\
\hline 10 & Pelargonic acid & $3.5 \pm 0.3$ & $3.2 \pm 0.7$ \\
\hline 16 & $\gamma$-Aminobutyric acid & $2.2 \pm 1.2$ & $4.7 \pm 1.1$ \\
\hline 17 & $2,3,4$-Trihydroxybutyric acid & $0.3 \pm 0.3$ & $0.4 \pm 0.4$ \\
\hline 20 & Lauric acid & $1.7 \pm 1.7$ & $6.5 \pm 5.6$ \\
\hline
\end{tabular}

TCA: Tricarboxylic acid. aAs indicated in Figure 3. Data are the mean \pm SE $(n=3)$. *Significantly different at $p<0.05$ (one-way ANOVA followed by post-hoc Tukey-Kramer test, vs. CS under normoxia).

signaling, EMT, p53 expression, and activating caspase-3. Glycine was discovered to be an indicator of the cellular state in hypoxic CS treated with $\mathrm{FxOH}$. This is the first report demonstrating suppression of sphere formation in CS under hypoxic conditions by $\mathrm{FxOH}$ with a synchronizing metabolite alteration.

We initially examined the CCSC properties of HT-29 CS under hypoxic conditions. To this end, HIF1 $\alpha$ and $\beta$-catenin expression, cell surface markers, EMT phenotype, and sphere-reforming capacity were investigated. Under hypoxic conditions, expression of HIF1 $\alpha$ (a marker of hypoxia) increased in CS, and that CD44v and CD44s (CCSC marker) decreased compared with normoxia. In addition, EpCAM (a CCSC marker) and E-cadherin, $N$-cadherin and vimentin (EMT-related proteins) did not show significant changes between hypoxic and normoxic conditions. Sphere-reforming activities were also approximately maintained between the two conditions (Figure 2A-C). CD44 glycoprotein, a hyaluronan receptor, is known to play an essential role in tumor cell adhesion, invasion and metastasis by binding to a

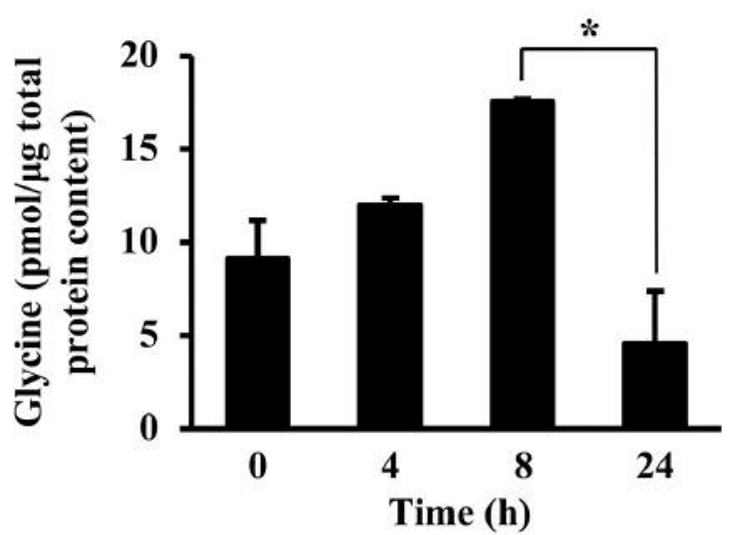

Figure 7. Time-dependent change of glycine by fucoxanthinol ( $\mathrm{FxOH}$ ) treatment in colonospheres (CS) formed from HT-29 cells under hypoxic conditions. CS formed from HT-29 cells for 2 days under hypoxic conditions were treated with $50 \mu \mathrm{M}$ of $\mathrm{FxOH}$ for an additional 4, 8 and $24 \mathrm{~h}$ under subsequent hypoxic conditions. The CS were collected and intracellular glycine content was analyzed by gas chromatography-mass spectrometry (GC-MS). GC-MS conditions are given in the Materials and Methods section. Values are means $\pm S E(n=3)$. *Significantly different at $p<0.05$ by one-way ANOVA with post-hoc Tukey-Kramer test.

pericellular matrix of hyaluronan in CRC cells $(26,27)$. The level of CD44 expression is reported to be positively associated with CRC progression and with the stemness function of CCSC (28). We previously confirmed that HT-29 CS formed from adherent parent cells (PCs) normoxia enhanced CD44v, EpCAM, and vimentin expression, and sphere forming activity $(25, \mathrm{M}$. Terasaki et al., unpublished data). Therefore, it was suggested that the CCSC properties of hypoxic CS would be enhanced compared with parental cells. There is little literature available about HIF1 $\alpha$ expression, its molecular and metabolic regulation in HT-29 CS or other CCSC models under hypoxic conditions and normoxic conditions. It has been demonstrated that CCSClike clonogenicity for HT-29 cells grown in matrigel under hypoxic conditions were enhanced in comparison with those under normoxic conditions (5). In adherent cell-type as nonCCSC phenotype, some researchers revealed that under hypoxic conditions CRC cells increased expression of cyclooxygenase- 2 and vimentin, and induced extracellular signal-regulated kinase activation and underwent $G_{0} / G_{1}$ arrest $(29,30)$. There are inconsistencies between those previous studies and the present study, we used HT-29 CS under hypoxic conditions as a hypoxia-like CS model.

Oxygen deprivation of HT-29 CS resulted in metabolic reprogramming, in which the glycine level was significantly reduced, and the levels of almost all other metabolites detected also declined, but without statistical significance (Figure 3 and Table I). Among amino acids, aspartate, serine, 
Table II. Metabolite profiles in colonospheres treated with fucoxanthinol ( $\mathrm{FxOH}$ ) under hypoxic and normoxic conditions.

\begin{tabular}{|c|c|c|c|c|c|}
\hline \multirow{3}{*}{$\begin{array}{l}\text { Peak } \\
\text { no. }{ }^{\text {a }}\end{array}$} & \multirow[t]{3}{*}{ Compound } & \multicolumn{4}{|c|}{ Metabolite, $\mathrm{pmol} / \mu \mathrm{g}$ total protein content } \\
\hline & & \multicolumn{2}{|c|}{ Hypoxia } & \multicolumn{2}{|c|}{ Normoxia } \\
\hline & & Control & $\mathrm{FxOH}$ & Control & $\mathrm{FxOH}$ \\
\hline & Amino acids & & & & \\
\hline 3 & Valine & ND & ND & $0.6 \pm 0.6$ & $1.1 \pm 1.1$ \\
\hline 5 & Leucine & $1.3 \pm 1.3$ & ND & $2.7 \pm 1.4$ & ND \\
\hline 6 & Proline & $4.8 \pm 4.0$ & ND & $10.5 \pm 1.5$ & $1.4 \pm 1.2 *$ \\
\hline 7 & Glycine & $6.7 \pm 1.6$ & ND* & $15.5 \pm 0.7$ & $5.3 \pm 2.8^{*}$ \\
\hline 11 & Alanine & $0.4 \pm 0.4$ & ND & $0.5 \pm 0.5$ & ND \\
\hline 12 & Serine & ND & ND & $3.7 \pm 0.5$ & $0.6 \pm 0.4 *$ \\
\hline 13 & Threonine & $3.2 \pm 1.2$ & $1.3 \pm 1.3$ & $10.1 \pm 1.3$ & $2.4 \pm 0.9^{*}$ \\
\hline 15 & Aspartic acid & $1.8 \pm 0.8$ & ND & $4.6 \pm 0.4$ & $0.7 \pm 0.7^{*}$ \\
\hline 18 & Glutamic acid & $3.4 \pm 1.3$ & ND & $15.9 \pm 2.5$ & $1.7 \pm 1.1^{*}$ \\
\hline \multirow[t]{2}{*}{19} & Phenylalanine & ND & ND & $1.5 \pm 0.8$ & ND \\
\hline & Dicarboxylic acids (TCA cycle) & & & & \\
\hline 8 & Succinic acid & $0.4 \pm 0.4$ & ND & $1.5 \pm 0.1$ & ND* \\
\hline 9 & Fumaric acid & ND & ND & $0.9 \pm 0.7$ & ND \\
\hline \multirow[t]{2}{*}{14} & Malic acid & $1.4 \pm 0.9$ & ND & $5.1 \pm 0.9$ & $4.0 \pm 2.5$ \\
\hline & Carboxylic acids/ other & & & & \\
\hline 1 & Pyruvic acid & ND & ND & $1.6 \pm 0.8$ & $0.6 \pm 0.4$ \\
\hline 2 & Propionic acid & $99.8 \pm 45.5$ & $104.4 \pm 14.0$ & $111.6 \pm 24.3$ & $95.4 \pm 21.6$ \\
\hline 4 & Butyric acid & $26.4 \pm 5.7$ & $20.9 \pm 9.9$ & $21.8 \pm 1.3$ & $16.9 \pm 2.7$ \\
\hline 10 & Pelargonic acid & $3.2 \pm 1.4$ & $1.6 \pm 1.6$ & $2.4 \pm 1.4$ & $0.5 \pm 0.5$ \\
\hline 16 & $\gamma$-Aminobutyric acid & $3.6 \pm 1.3$ & $3.1 \pm 3.1$ & $5.0 \pm 0.8$ & $4.1 \pm 1.4$ \\
\hline 17 & $2,3,4$-Trihydroxybutyric acid & ND & ND & $1.9 \pm 0.1$ & $0.7 \pm 0.7$ \\
\hline 20 & Lauric acid & $9.2 \pm 5.0$ & $10.1 \pm 1.2$ & $5.1 \pm 2.4$ & $8.6 \pm 3.8$ \\
\hline
\end{tabular}

ND: Metabolite not detected at a level exceeding the signal/noise ratio (3:1). TCA: Tricarboxylic acid. a As indicated in Figure 5. Data are the mean \pm SE $(n=3)$. *Significantly different at $\mathrm{p}<0.05$ (one-way ANOVA followed by post-hoc Tukey-Kramer test, $v s$. CS under normoxia).

glutamic acid and glutamine were demonstrated to be targets for cancer therapeutics (31-36). It is interesting that these amino acids are known to contribute to glycolysis and mitochondrial respiration. However, to our knowledge, this is the only report that demonstrates the cellular metabolism of CCSC under hypoxia.

FxOH significantly inhibited sphere formation under both hypoxia and normoxia CS to the same degree (Figure 4). FxOH treatment began to suppress PPAR $\gamma$ at $4 \mathrm{~h}$, and reduced levels of integrin, and proteins involved in MAPK, WNT and STAT signaling, and in EMT were observed from $8 \mathrm{~h}$ to $24 \mathrm{~h}$. At $24 \mathrm{~h}$, caspase- 3 activation and HIF1 $\alpha$ and p53 depression were observed in hypoxic CS (Figure 6). Interestingly, these time-dependent molecular changes induced by $\mathrm{FxOH}$ were almost the same under hypoxic and normoxic conditions, as shown in our previous study (M. Terasaki et al., unpublished data). Our findings suggest that the molecular mechanisms involved in CS apoptosis induced by $\mathrm{FxOH}$ may not be affected by oxygen concentration.

Regarding other carotenoids, $\beta$-carotene attenuated CSC markers, HIF1 $\alpha$ and vascular endothelial growth factor
(VEGF) in a xenograft mouse model using neuroblastoma SK$\mathrm{N}-\mathrm{BE}(2) \mathrm{C}$ cells (37). Lycopene-enriched tomato extract suppressed HIF1 $\alpha$, VEGF and matrix metalloproteinases in a mouse model of $N$-nitrosodiethylamine-induced hepatocellular cancer (38). Retinoic acid induced differentiation through down-regulation of HIF1 $\alpha$ and up-regulation of neurofilament $\mathrm{M}(\mathrm{NF}-\mathrm{M})$ and neuron-specific nuclear protein $(\mathrm{Neu}-\mathrm{N})$ in human neuroblastoma NB1691 cells (39).

Compared with untreated control CS, most of the metabolite changes in $\mathrm{CS}$ treated with $\mathrm{FxOH}$ were the same between hypoxic conditions and normoxic conditions (Figure 5 and Table II). However, the limitation of this is that the detectable levels of metabolite in CS under hypoxic conditions were less than those of CS under normoxic conditions. Nevertheless, FxOH treatment significantly reduced the level of glycine in hypoxia $\mathrm{CS}$ formed from 8 to 24 h compared with untreated control CS (Figure 7). $\mathrm{FxOH}$ may particularly attenuate serine biosynthesis and modulate the glutathione (GSH)/glutathione disulfide (GSSG) redox system in internal and external mitochondria in CS. The changes in mitochondria function by fucoxanthin or $\mathrm{FxOH}$ 
treatment observed under normoxic conditions have been reported elsewhere (40, M. Terasaki et al.; unpublished data). To our knowledge, our report is the first to show the alteration of metabolites by carotenoids in CSCs or CSC-like spheroids under hypoxic conditions.

In summary, $\mathrm{FxOH}$ attenuated the sphere formation of $\mathrm{CS}$ derived from HT-29 human CRC cells under hypoxia, in part, by suppressing HIF1 $\alpha$ expression and some signal transduction. A change in glycine level was detected as a metabolite marker of the suppression of sphere formation in CS under hypoxia. Further studies are needed to confirm that glycine is a good marker reflecting the state of hypoxia of CCSCs not only in in vitro models, but also in humans after fucoxanthin or $\mathrm{FxOH}$ administration.

\section{Conflicts of Interest}

None.

\section{Acknowledgements}

This work was supported in part by JSPS KAKENHI Grant Number 16K07880.

\section{References}

1 Ferlay J, Soerjomataram I, Dikshit R, Eser S, Mathers C, Rebelo M, Parkin DM, Forman D and Bray F: Cancer incidence and mortality worldwide: source, methods and major patterns in GLOBOCAN 2012. Int J Cancer 136: E359-E386, 2015.

2 World Health Organization: Diet, Nutrition and the Prevention of Chronic Diseases: Report of a Joint WHO/FAO Expert Consultation. Geneva: World Health Organization Publishers, Inc., pp. 95-104, 2003.

3 Dalerba P, Dylla SJ, Park IK, Liu R, Wang X, Cho RW, Hoey Y, Gurney A, Huang EH, Simeone DM, Shelton AA, Parmiani G, Castelli $\mathrm{C}$ and Clarke MF: Phenotypic characterization of human colorectal cancer stem cells. Proc Natl Acad Sci 104: 1015810163, 2007.

4 Vermeulen L, Todaro M, de Sousa Mello F, Sprick MR, Kemper K, Perez Alea M, Richel DJ, Stassi G and Medema JP: Single-cell cloning of colon cancer stem cells reveals a multi-lineage differentiation capacity. Proc Natl Acad Sci 105: 13427-13432, 2008.

5 Yeung TM, Gandhi SC and Bodmer WF: Hypoxia and lineage specification of cell line-derived colorectal cancer stem cells. Proc Natl Acad Sci USA 108: 4382-4387, 2011.

6 Santoyo-Ramos P, Likhatcheva M, García-Zepeda EA, Castañeda-Patlán MC and Robles-Flores M: Hypoxia-inducible factors modulate the stemness and malignancy of colon cancer cells by playing opposite roles in canonical Wnt signaling. PLoS One 14: e112580, 2014.

7 Nagaraju GP, Bramhachari PV, Raghu G and EI-Rayes BF: Hypoxia-inducible factor- $1 \alpha$ : Its role in colorectal carcinogenesis and metastasis. Cancer Lett 366: 11-18, 2015.

8 Gordan JD, Thompson CB and Simon MC: HIF and c-Myc: sibling rivals for control of cancer cell metabolism and proliferation. Cancer Cell 12: 108-113, 2007.
9 Tsuzuki Y, Fukumura D, Oosthuyse B, Koike C, Carmeliet P, Jain RK: Vascular endothelial growth factor (VEGF) modulation by targeting hypoxia-inducible factor-1alpha --> hypoxia response element --> VEGF cascade differentially regulates vascular response and growth rate in tumors. Cancer Res 60: 6248-6252, 2000.

10 Koshiji M, Kageyama Y, Pete EA, Horikawa I, Barrett JC and Huang LE: HIF1alpha induces cell-cycle arrest by functionally counteracting Myc. EMBO J 23: 1949-1956, 2004.

11 D'Ignazio L, Batie M and Rocha S: Hypoxia and Inflammation in cancer, focus on HIF and NF-kB. Biomedicines 5: 21-43, 2017.

12 Yoshida M, Hatano N, Nishiumi S, Irino Y, Izumi Y, Takenawa $\mathrm{T}$ and Azuma T: Diagnosis of gastroenterological diseases by metabolome analysis using gas chromatography-mass spectrometry. J Gastroenterol 47: 9-20, 2012.

13 Dazard JE, Sandlers Y, Doerner SK, Berger NA and Brunengraber H: Metabolomics of $\mathrm{Apc}^{\mathrm{Min} /+}$ mice genetically susceptible to intestinal cancer. BMC Syst Biol 8: 72, 2014.

14 Yoshie T, Nishiumi S, Izumi Y, Sakai A, Inoue J, Azuma T and Yoshida M: Regulation of the metabolite profile by an $A P C$ gene mutation in colorectal cancer. Cancer Sci 103: 1010-1021, 2012.

15 Terasaki M, Hirose A, Narayan B, Baba Y, Kawagoe C, Yasui H, Saga N, Hosokawa M and Miyashita K: Evaluation of recoverable functional lipid components of several brown seaweeds (phaeophyta) from Japan with special reference to fucoxanthin and fucosterol contents. J Phycol 45: 974-980, 2009.

16 Terasaki M, Narayan B, Kamogawa H, Nomura M, Stephen NM, Kawagoe C, Hosokawa M and Miyashita K: Carotenoid profile of edible Japanese seaweeds: An improved HPLC method for separation of major carotenoids. J Aquatic Food Prod Tech 21: 468-479, 2012.

17 Kim JM, Araki S, Kim DJ, Park CB, Takasuka N, BabaToriyama H, Ota T, Nir Z, Khachik F, Shimidzu N, Tanaka Y, Osawa T, Uraji T, Murakoshi M, Nishino H and Tsuda H: Chemopreventive effects of carotenoids and curcumins on mouse colon carcinogenesis after 1,2-dimethylhydrazine initiation. Carcinogenesis 19: 81-85, 1998.

18 Nishino H, Murakoshi M, Tokuda H and Satomi Y: Cancer prevention by carotenoids. Arch Biochem Biophys 483: 165-168, 2009.

19 Hosokawa M, Kudo M, Maeda H, Kohno H, Tanaka T and Miyashita K: Fucoxanthin induces apoptosis and enhances the antiproliferative effect of the PPARgamma ligand, troglitazone, on colon cancer cells. Biochim Biophys Acta 1675: 113-119, 2004.

20 Das SK, Hashimoto T, Shimizu K, Yoshida T, Sakai T, Sowa Y, Komoto A and Kanazawa K: Fucoxanthin induces cell cycle arrest at $\mathrm{G}_{0} / \mathrm{G}_{1}$ phase in human colon carcinoma cells through up-regulation of $\mathrm{p} 21^{\mathrm{WAK} 1 / \mathrm{CIP} 1}$. Biochim Biophys Acta 1726: 328-335, 2005.

21 Takahashi K, Hosokawa M, Kasajima H, Hatanaka K, Kudo K, Shimoyama $\mathrm{N}$ and Miyashita K: Anticancer effects of fucoxanthin and fucoxanthinol on colorectal cancer cell lines and colorectal cancer tissues. Oncol Lett 10: 1463-1467, 2015.

22 Asai A, Yonekura L and Nagao A: Low bioavailability of dietary epoxyxanthophylls in humans. Br J Nutr 100: 273-277, 2008.

23 Hashimoto T, Ozaki Y, Mizuno M, Yoshida M, Nishitani Y, Azuma $\mathrm{T}$, Komoto A, Maoka $\mathrm{T}$, Tanino $\mathrm{Y}$ and Kanazawa $\mathrm{K}$ : Pharmacokinetics of fucoxanthinol in human plasma after the oral administration of kombu extract. Br J Nutr 107: 1566-1569, 2012. 
24 Kanwar SS, Yu Y, Nautiyal J, Patel BB and Majumdar AP: The $\mathrm{Wnt} /$ beta-catenin pathway regulates growth and maintenance of colonospheres. Mol Cancer 9: 212-225, 2010.

25 Terasaki M, Maeda H, Miyashita K, Tanaka T, Miyamoto S and Mutoh M: A marine bio-functional lipid, fucoxanthinol, attenuates human colorectal cancer stem-like cell tumorigenicity and sphere formation. J Clin Biochem Nutr 61: 25-32, 2017.

26 Isacke $\mathrm{CM}$ and Yarwood $\mathrm{H}$ : The hyaluronan receptor, CD44. Int J Biochem Cell Biol 34: 718-721, 2002.

27 Bánkey B, Rásó-Barnett L, Barbai T, Tímár J, Becságh P and Rásó E: Characteristics of CD44 alternative splice pattern in the course of human colorectal adenocarcinoma progression. Mol Cancer 11: 83-97, 2012.

28 Zeilstra J, Joosten SP, Dokter M, Verwiel E, Spaargaren M and Pals ST: Deletion of the WNT target and cancer stem cell marker CD44 in $\operatorname{Apc}(\mathrm{Min} /+)$ mice attenuates intestinal tumorigenesis. Cancer Res 68: 3655-3661, 2008.

29 Kaidi A, Qualtrough D, Williams AC and Paraskeva C: Direct transcriptional up-regulation of cyclooxygenase-2 by hypoxiainducible factor (HIF)-1 promotes colorectal tumor cell survival and enhances HIF1 transcriptional activity during hypoxia. Cancer Res 66: 6683-6691, 2006.

30 Krishnamachary B, Berg-Dixon S, Kelly B, Agani F, Feldser D, Ferreira G, Iyer N, LaRusch J, Park B, Taghavi P and Semenza GL: Regulation of colon carcinoma cell invasion by hypoxiainducible factor 1. Cancer Res 63: 1138-1143, 2003.

31 Warburg O: On the origin of cancer cells. Science 123: 309-314, 1956.

32 Sato M, Kawana K, Adachi K, Fujimoto A, Yoshida M, Nakamura $\mathrm{H}$, Nishida $\mathrm{H}$, Inoue $\mathrm{T}$, Taguchi A, Takahashi $\mathrm{J}$, Eguchi S, Yamashita A, Tomio K, Wada-Hiraike O, Oda K, Nagamatsu T, Osuga Y and Fujii T: Spheroid cancer stem cells display reprogrammed metabolism and obtain energy by actively running the tricarboxylic acid (TCA) cycle. Oncotarget 7: 33297-33305, 2016.

33 Vermeersch KA, Wang L, Mezencev R, McDonald JF and Styczynski MP: OVCAR-3 spheroid-derived cells display distinct metabolic profiles. PLos One 10: e0118262, 2015.
34 Penkert J, Ripperger T, Schieck M, Schiegelberger B, Steinemann D and Illig T: On metabolic reprogramming and tumor biology: A comprehensive survey of metabolism in breast cancer. Oncotarget 7: 67626-67649, 2016.

35 Lin SH, Liu T, Ming X, Tang Z, Fu L, Schmitt-Kopplin P, Kanawati B, Guan XY and Cai Z: Regulatory role of hexosamine biosynthetic pathway on hepatic cancer stem cell marker CD133 under low glucose conditions. Sci Rep 6: 21184, 2016.

36 Chen KY, Liu X, Bu P, Lin CS, Rakhilin N, Locasale JW and Shen X: A metabolic signature of colon cancer-initiating cells. Conf Proc IEEE Eng Med Biol Soc 2014: 4759-4762, 2014.

37 Lim JY, Kim YS, Kim KM, Min SJ and Kim Y: $\beta$-Carotene inhibits neuroblastoma tumorigenesis by regulating cell differentiation and cancer cell stemness. Biochem Biophys Res Commun 450: 1475-1480, 2014.

38 Bhatia N, Gupta P, Singh B and Koul A: Lycopene enriched tomato extract inhibits hypoxia, angiogenesis, and metastatic markers in early-stage $N$-nitrosodiethylamine-induced hepatocellular carcinoma. Nutr Cancer 67: 1268-1275, 2015.

39 Bhaskara VK, Mohanam I, Rao JS and Mohanam S: Intermittent hypoxia regulates stem-like characteristics and differentiation of neuroblastoma cells. PLoS One 7: e30905, 2012.

40 Kotake-Nara E, Terasaki M and Nagao A: Characterization of apoptosis induced by fucoxanthin in human promyelocytic leukemia cells. Biosci Biotechnol Biochem 69: 224-227, 2005.

Received January 31, 2018

Revised February 25, 2018

Accepted February 27, 2018 